\title{
Iterative Algorithm for Solving a Class of Quaternion Matrix Equation over the Generalized $(P, Q)$-Reflexive Matrices
}

\author{
Ning $\mathrm{Li}^{1}$ and Qing-Wen Wang ${ }^{2}$ \\ ${ }^{1}$ School of Mathematics and Quantitative Economics, Shandong University of Finance and Economics, Jinan, \\ Shandong Province 250002, China \\ ${ }^{2}$ Department of Mathematics, Shanghai University, Shanghai 200444, China
}

Correspondence should be addressed to Qing-Wen Wang; wqw369@yahoo.com

Received 18 April 2013; Accepted 12 August 2013

Academic Editor: Douglas Anderson

Copyright (C) 2013 N. Li and Q.-W. Wang. This is an open access article distributed under the Creative Commons Attribution License, which permits unrestricted use, distribution, and reproduction in any medium, provided the original work is properly cited.

\begin{abstract}
The matrix equation $\sum_{l=1}^{u} A_{l} X B_{l}+\sum_{s=1}^{v} C_{s} X^{T} D_{s}=F$, which includes some frequently investigated matrix equations as its special cases, plays important roles in the system theory. In this paper, we propose an iterative algorithm for solving the quaternion matrix equation $\sum_{l=1}^{u} A_{l} X B_{l}+\sum_{s=1}^{v} C_{s} X^{T} D_{s}=F$ over generalized $(P, Q)$-reflexive matrices. The proposed iterative algorithm automatically determines the solvability of the quaternion matrix equation over generalized $(P, Q)$-reflexive matrices. When the matrix equation is consistent over generalized $(P, Q)$-reflexive matrices, the sequence $\{X(k)\}$ generated by the introduced algorithm converges to a generalized $(P, Q)$-reflexive solution of the quaternion matrix equation. And the sequence $\{X(k)\}$ converges to the least Frobenius norm generalized $(P, Q)$-reflexive solution of the quaternion matrix equation when an appropriate initial iterative matrix is chosen. Furthermore, the optimal approximate generalized $(P, Q)$-reflexive solution for a given generalized $(P, Q)$-reflexive matrix $X_{0}$ can be derived. The numerical results indicate that the iterative algorithm is quite efficient.
\end{abstract}

\section{Introduction and Notations}

Quaternion matrix equation is one of the topics of very active research in matrix theory and applications, and some important results have been developed. For example, Kyrchei [1] derived the explicit representation formulas for the minimum norm least squares solutions of quaternion matrix equations $A X=B, X A=B, A X B=D$. Yuan and Liao [2] derived the expressions of the least squares ( $j$-self-conjugate) solution with the least norm of the quaternion matrix equation $X-$ $A \widehat{X} B=C$. Song et al. [3] obtained the expressions of the explicit solutions of quaternion matrix equations $X F-A X=$ $B Y$ and $X F-A \widetilde{X}=B Y$. He and Wang [4] studied the necessary and sufficient conditions for the existence of a solution to the quaternion matrix equation $A_{1} X+\left(A_{1} X\right)^{\eta *}+$ $B_{1} Y\left(B_{1}\right)^{\eta *}+C_{1} Z\left(C_{1}\right)^{\eta *}=D_{1}$ and derived a general solution when the equation is solvable.
The definition of generalized $(P, Q)$-reflexive matrix can be found in [5]. A complex matrix $A$ is called $(P, Q)$-generalized reflexive (generalized anti-reflexive) if $A=P A Q(A=$ $-P A Q)$, where $P$ and $Q$ are two generalized reflection matrices; that is, $P=P^{H}=P^{-1}$ and $Q=Q^{H}=Q^{-1}$. The generalized $(P, Q)$-reflexive matrices have been widely used in engineering and scientific computations [5-7]. Throughout, we use $\mathbb{\boxplus}_{r}^{m \times n}(P, Q)$ to denote the set of $m \times n$ generalized $(P, Q)$ reflexive quaternion matrices.

The iterative method is a very important method to solve matrix equations. Peng et al. $[8,9]$ constructed iteration methods to solve the symmetric and reflexive solutions of matrix equations $A_{1} X B_{1}=C_{1}, A_{2} X B_{2}=C_{2}$. Ding and Chen $[10,11]$ developed iterative methods for solving a wide variety of matrix equations, based on the hierarchical identification principle [12]. Peng [13-15] presented several efficient iterative methods to solve the constrained least squares solutions 
of linear matrix equations $A X B=C$ and $A X B+C Y D=E$, by using Paige's algorithm [16] as the frame method. Wang et al. [17] proposed iterative algorithms for solving the matrix equation $A X B+C X^{T} D=E$. Xie et al. [18] presented a gradient based iterative algorithm for solving

$$
\sum_{l=1}^{u} A_{l} X B_{l}+\sum_{s=1}^{v} C_{s} X^{T} D_{s}=F .
$$

In [19], Li et al. proposed iterative algorithms to solve the minimal norm least squares solution to (1). Duan et al. [20-24] proposed iterative algorithms for the (Hermitian) positive definite solutions of some nonlinear matrix equations. Dehghan and Hajarian constructed iterative algorithms to solve several linear matrix equations over (anti-)reflexive $[25,26]$, generalized centrosymmetric $[27,28]$, and generalized bisymmetric $[29,30]$ matrices. Recently, Wu et al. [31-35] proposed iterative algorithms for solving various complex matrix equations. Wang et al. [36] derived an iterative method for finding the minimum-norm solution of the QLS problem in quaternionic quantum theory.

However, to our best knowledge, the generalized $(P, Q)$ reflexive solution of (1) over the quaternion algebra $\mathbb{W}$ has not been considered so far. Due to the noncommutativity of $\mathbb{H}$, some well-known equalities for complex and real matrices no longer hold for quaternion matrices, which make the study of quaternion matrix equation more complex than that of real and complex equation. Motivated by the work mentioned previously and keeping the interests and wide applications of quaternion matrices in view (e.g., [37-47]), we, in this paper, consider an iterative method for the following two problems.

Problem 1. For given matrices $A_{l}, C_{s} \in \mathbb{H}^{p \times n}, B_{l}, D_{s} \in \mathbb{H}^{n \times q}$, $l=1,2, \ldots, u, s=1,2, \ldots, v, F \in \mathbb{H}^{p \times q}$, and the generalized reflection matrices $P \in \mathbb{H}^{n \times n}, Q \in \mathbb{H}^{n \times n}$, find that $X \in$ $\mathbb{M}_{r}^{n \times n}(P, Q)$, such that

$$
\sum_{l=1}^{u} A_{l} X B_{l}+\sum_{s=1}^{v} C_{s} X^{T} D_{s}=F .
$$

Problem 2. When Problem 1 is consistent, let its solution set be denoted by $S_{X}$. For a given $(P, Q)$-reflexive matrix $X_{0} \in$ $\mathbb{H}_{r}^{n \times n}(P, Q)$, find $\widehat{X} \in \mathbb{H}_{r}^{n \times n}(P, Q)$, such that

$$
\left\|\widehat{X}-X_{0}\right\|=\min _{X \in S_{X}}\left\|X-X_{0}\right\| \text {. }
$$

The matrix equation (2) plays important roles in the system theory [48-50]. Moreover, (2) obviously includes the matrix equations $A X B+C X^{T} D=F, A X+X^{T} D=F$, and $A X B=C$ as special cases, which have been investigated in $[17,28,36,51-53]$.

Throughout this paper, $\mathbb{R}^{m \times n}$ and $\mathbb{H}^{m \times n}$ represent the set of all $m \times n$ real matrices and the set of all $m \times n$ matrices over the quaternion algebra $\mathbb{U}=\left\{a_{1}+a_{2} i+a_{3} j+a_{4} k\right.$ । $\left.i^{2}=j^{2}=k^{2}=i j k=-1, a_{1}, a_{2}, a_{3}, a_{4} \in \mathbb{R}\right\}$. We use $A^{H}$, $A^{T}, \bar{A}, \operatorname{tr}(A), R(A), \operatorname{Re}(A)$, and $\operatorname{vec}(A)$ to denote the conjugate transpose, the transpose, the conjugate, the trace, the column space, the real part, and the $m n \times 1$ vector formed by the vertical concatenation of the respective columns of a matrix $A$; respectively. We use $\|A\|$ to denote the Frobenius norm of $A$, that is, $\|A\|=\sqrt{\operatorname{tr}\left(A^{H} A\right)}$. The Kronecker matrix product and Hadamard matrix product of the matrices $A$ and $B$ are denoted by $A \otimes B$ and $A \odot B$. We use $I$ to denote the identity matrix with the appropriate size.

\section{Preliminaries}

In this section, we provide some results which will play important roles in this paper.

Firstly, we recall the definition of real inner product space. Then we define a real inner product in the space $\mathbb{M}^{m \times n}$ over the field $\mathbb{R}$.

Definition 3 (see [54]). A real inner product space is a vector space $V$ over the real field $\mathbb{R}$ together with an inner product defined by a mapping

$$
\langle\cdot, \cdot\rangle: V \times V \rightarrow \mathbb{R}
$$

satisfying the following three axioms for all vectors $x, y, z \in$ $V$ and all scalars $a \in \mathbb{R}$.

(1) Symmetry: $\langle x, y\rangle=\langle y, x\rangle$;

(2) Linearity in the first argument: $\langle a x, y\rangle=a\langle x, y\rangle,\langle x+$ $y, z\rangle=\langle x, z\rangle+\langle y, z\rangle$

(3) Positive definiteness: $\langle x, x\rangle>0$ for all $x \neq 0$.

In [33-35], the following real inner product was presented to solve some complex matrix equations:

$$
\langle A, B\rangle=\operatorname{Re}\left[\operatorname{tr}\left(B^{H} A\right)\right],
$$

for $A, B \in \mathbb{C}^{m \times n}$. It is easy to verify that if we let $A, B \in \mathbb{T}^{m \times n}$, (5) also defines a real inner product in $\mathbb{H}^{m \times n}$ over $\mathbb{R}$. We denote this real inner product space by $\left(\mathbb{H}^{m \times n}, \mathbb{R},\langle\cdot, \cdot\rangle\right)$. From the equalities

$$
\begin{aligned}
\sqrt{\langle A, A\rangle} & =\sqrt{\operatorname{Re}\left[\operatorname{tr}\left(A^{H} A\right)\right]} \\
& =\sqrt{\operatorname{tr}\left(A^{H} A\right)}=\|A\|, \quad A \in \mathbb{H}^{m \times n},
\end{aligned}
$$

we know that the induced matrix norm by the introduced inner product $\langle\cdot, \cdot\rangle$ is exactly the Frobenius norm. For convenience, we still use $\|\cdot\|$ to denote the induced matrix norm. Let $E_{i j}$ denote the $m \times n$ matrix whose $(i, j)$ entry is 1 , and the other elements are zeros. In inner product space $\left(\mathbb{H}^{m \times n}, \mathbb{R},\langle\cdot, \cdot\rangle\right)$, it is easy to verify that $E_{i j}, E_{i j} i, E_{i j} j, E_{i j} k, i=$ $1,2, \ldots m, j=1,2, \ldots n$, is an orthonormal basis, which reveals that the dimension of the inner product space $\left(\mathbb{H}^{m \times n}, \mathbb{R},\langle\cdot, \cdot\rangle\right)$ is $4 m n$.

Next, we introduce the well-known real representation of a quaternion matrix. For an arbitrary quaternion matrix $M=$ $M_{1}+M_{2} i+M_{3} j+M_{4} k \in \mathbb{M}^{m \times n}$, a map $\phi(\cdot)$, from $\mathbb{H}^{m \times n}$ to $\mathbb{R}^{4 m \times 4 n}$, can be defined as

$$
\phi(M)=\left[\begin{array}{cccc}
M_{1} & -M_{2} & -M_{3} & -M_{4} \\
M_{2} & M_{1} & -M_{4} & M_{3} \\
M_{3} & M_{4} & M_{1} & -M_{2} \\
M_{4} & -M_{3} & M_{2} & M_{1}
\end{array}\right] .
$$


Let $M$ and $N$ be two arbitrary quaternion matrices with appropriate size. We know that $\phi(\cdot)$ satisfies the following properties:

$$
\begin{gathered}
M=N \Longleftrightarrow \phi(M)=\phi(N), \\
\phi(M+N)=\phi(M)+\phi(N), \\
\phi(M N)=\phi(M) \phi(N), \\
\phi(k M)=k \phi(M), \quad k \in \mathbb{R}, \\
\phi\left(M^{H}\right)=\phi^{T}(M) .
\end{gathered}
$$

It follows a simple verification that $\phi(\cdot)$ also satisfies the following two properties which is useful for some deduction in this paper:

$$
\begin{gathered}
\|\phi(M)\|=2\|M\|, \\
\phi\left(M^{T}\right)=2 \phi^{T}(M) \odot W-\phi^{T}(M),
\end{gathered}
$$

where

$$
W=\left[\begin{array}{llll}
E & 0 & 0 & 0 \\
0 & E & 0 & 0 \\
0 & 0 & E & 0 \\
0 & 0 & 0 & E
\end{array}\right]
$$

and $E$ is a matrix whose elements are all equal to one with the same size as $M$.

Finally, we recall some results about the commutation matrix. A commutation matrix $P(m, n)$ is a $m n \times m n$ matrix which has the following explicit form:

$$
P(m, n)=\sum_{i=1}^{m} \sum_{j=1}^{n} E_{i j} \otimes E_{i j}^{T}=\left[E_{i j}^{T}\right]_{\substack{i=1, \ldots, m \\ j=1, \ldots, n}}
$$

Moreover, $P(m, n)$ is a permutation matrix and $P(m, n)=$ $P^{T}(n, m)=P^{-1}(n, m)$. We have the following lemmas on the commutation matrix.

Lemma 4 (see [55]). Let $A$ be an $m \times n$ matrix. There is a commutation matrix $P(m, n)$ such that

$$
\operatorname{vec}\left(A^{T}\right)=P(m, n) \operatorname{vec}(A) .
$$

Lemma 5 (see [55]). Let $A$ be an $m \times n$ matrix and $B$ a $p \times q$ matrix. There exist two commutation matrices $P(m, p)$ and $P(n, q)$ such that

$$
B \otimes A=P^{T}(m, p)(A \otimes B) P(n, q) .
$$

\section{An Iterative Algorithm for Solving Problem 1}

In this section, we firstly construct an algorithm for solving Problem 1. Then we show that if the problem is consistent, the sequence $\{X(k)\}$ generated by the introduced algorithm converges to a solution of Problem 1 within at most $4 p q$ iteration steps.
Algorithm 6. (1) Input matrices $A_{l}, C_{s} \in \mathbb{M}^{p \times n}, B_{l}, D_{s} \in$ $\mathbb{H}^{n \times q}, l=1,2, \ldots, u, s=1,2, \ldots, v, F \in \mathbb{T}^{p \times q}$, generalized reflection matrices $P \in \mathbb{H}^{n \times n}, Q \in \mathbb{H}^{n \times n}, X(1) \in \mathbb{H}_{r}^{n \times n}(P, Q)$.

(2) Calculate

$$
\begin{gathered}
R(1)=F-\sum_{l=1}^{u} A_{l} X(1) B_{l}-\sum_{s=1}^{v} C_{s} X^{T}(1) D_{s} ; \\
S(1)=\frac{1}{2}\left(\sum_{l=1}^{u} A_{l}^{H} R(1) B_{l}^{H}+\sum_{s=1}^{v}\left(C_{s}^{H} R(1) D_{s}^{H}\right)^{T}\right. \\
\left.+\sum_{l=1}^{u} P A_{l}^{H} R(1) B_{l}^{H} Q+\sum_{s=1}^{v} P\left(C_{s}^{H} R(1) D_{s}^{H}\right)^{T} Q\right) ; \\
T(1)=S(1) \\
k:=1 .
\end{gathered}
$$

(3) If $R(k)=0$, or $R(k) \neq 0$ and $T(k)=0$, stop; otherwise $k:=k+1$.

(4) Calculate

$$
X(k)=X(k-1)+\frac{\|R(k-1)\|^{2}}{\|T(k-1)\|^{2}} T(k-1) ;
$$$$
R(k)=R(k-1)
$$$$
-\frac{\|R(k-1)\|^{2}}{\|T(k-1)\|^{2}}\left(\sum_{l=1}^{u} A_{l} T(k-1) B_{l}\right.
$$$$
\left.+\sum_{s=1}^{v} C_{s} T^{T}(k-1) D_{s}\right)
$$

$$
\begin{aligned}
S(k)=\frac{1}{2}\left(\sum_{l=1}^{u} A_{l}^{H} R(k) B_{l}^{H}+\sum_{s=1}^{v}\left(C_{s}^{H} R(k) D_{s}^{H}\right)^{T}\right. \\
+\sum_{l=1}^{u} P A_{l}^{H} R(k) B_{l}^{H} Q \\
\left.+\sum_{s=1}^{v} P\left(C_{s}^{H} R(k) D_{s}^{H}\right)^{T} Q\right) \\
T(k)=S(k)+\frac{\|R(k)\|^{2}}{\|R(k-1)\|^{2}} T(k-1) .
\end{aligned}
$$

(5) Go to Step (3).

The following Lemmas show some properties of Algorithm 6. 
Lemma 7. The sequences $\{R(i)\},\{S(i)\}$, and $\{T(i)\}$ generated by Algorithm 6 satisfy

$$
\begin{aligned}
& \operatorname{Re}\left\{\operatorname{tr}\left[R^{H}(j+1) R(i)\right]\right\} \\
&= \operatorname{Re}\left\{\operatorname{tr}\left[R^{H}(j) R(i)\right]\right\} \\
&-\frac{\|R(j)\|^{2}}{\|T(j)\|^{2}} \operatorname{Re}\left\{\operatorname{tr}\left[T^{H}(j) S(i)\right]\right\}, \\
& \operatorname{Re}\left\{\operatorname{tr}\left[T^{H}(j+1) T(i)\right]\right\} \\
&= \frac{\|R(j+1)\|^{2}}{\|R(j)\|^{2}} \operatorname{Re}\left\{\operatorname{tr}\left[T^{H}(j) T(i)\right]\right\} \\
&+ \frac{\|T(i)\|^{2}}{\|R(i)\|^{2}}\left(\operatorname{Re}\left\{\operatorname{tr}\left[R^{H}(j+1) R(i)\right]\right\}\right. \\
&\left.-\operatorname{Re}\left\{\operatorname{tr}\left[R^{H}(j+1) R(i+1)\right]\right\}\right), \\
& \quad \text { for } i, j=1,2, \ldots .
\end{aligned}
$$

Proof. One has the following:

$$
\begin{aligned}
& \operatorname{Re}\left\{\operatorname{tr}\left[R^{H}(j+1) R(i)\right]\right\} \\
& =\operatorname{Re}\left\{\operatorname { t r } \left[\left(R(j)-\frac{\|R(j)\|^{2}}{\|T(j)\|^{2}}\right.\right.\right. \\
& \times\left(\sum_{l=1}^{u} A_{l} T(j) B_{l}\right. \\
& \left.\left.\left.\left.+\sum_{s=1}^{v} C_{s} T^{T}(j) D_{s}\right)\right)^{H} R(i)\right]\right\} \\
& =\operatorname{Re}\left\{\operatorname{tr}\left[R^{H}(j) R(i)\right]\right\}-\frac{\|R(j)\|^{2}}{\|T(j)\|^{2}} \\
& \quad \times \operatorname{Re}\left\{\operatorname { t r } \left[\sum_{l=1}^{u} B_{l}^{H} T^{H}(j) A_{l}^{H} R(i)\right.\right. \\
& \left.\left.+\sum_{s=1}^{v} D_{s}^{H} \frac{T(j)}{C_{s}^{H} R(i)}\right]\right\} \\
& =\operatorname{Re}\left\{\operatorname{tr}\left[R^{H}(j) R(i)\right]\right\}-\frac{\|R(j)\|^{2}}{\|T(j)\|^{2}} \\
& \quad \times \operatorname{Re}\left\{\operatorname { t r } \left[T ^ { H } ( j ) \left(\sum_{l=1}^{u} A_{l}^{H} R(i) B_{l}^{H}\right.\right.\right. \\
& \left.\left.\left.+\sum_{s=1}^{v}\left(C_{s}^{H} R(i) D_{s}^{H}\right)^{T}\right)\right]\right\}
\end{aligned}
$$

$$
\begin{gathered}
=\operatorname{Re}\left\{\operatorname{tr}\left[R^{H}(j) R(i)\right]\right\}-\frac{\|R(j)\|^{2}}{\|T(j)\|^{2}} \\
\times \operatorname{Re}\left\{\operatorname { t r } \left[T^{H}(j)\right.\right. \\
\times\left(\sum_{l=1}^{u} A_{l}^{H} R(i) B_{l}^{H}\right. \\
+\sum_{s=1}^{v}\left(C_{s}^{H} R(i) D_{s}^{H}\right)^{T} \\
+\sum_{l=1}^{u} P A_{l}^{H} R(i) B_{l}^{H} Q \\
\left.+\sum_{s=1}^{v} P\left(C_{s}^{H} R(i) D_{s}^{H}\right)^{T} Q\right) \\
\left.\left.\times 2^{-1}\right]\right\} \\
=\operatorname{Re}\left\{\operatorname{tr}\left[R^{H}(j) R(i)\right]\right\} \\
-\frac{\|R(j)\|^{2}}{\|T(j)\|^{2}} \operatorname{Re}\left\{\operatorname{tr}\left[T^{H}(j) S(i)\right]\right\} .
\end{gathered}
$$

Therefore, the conclusion (19) holds. Consider

$$
\begin{gathered}
\operatorname{Re}\left\{\operatorname{tr}\left[T^{H}(j+1) T(i)\right]\right\} \\
=\operatorname{Re}\left\{\operatorname{tr}\left[\left(S^{H}(j+1)+\frac{\|R(j+1)\|^{2}}{\|R(j)\|^{2}} T^{H}(j)\right) T(i)\right]\right\} \\
=\frac{\|R(j+1)\|^{2}}{\|R(j)\|^{2}} \operatorname{Re}\left\{\operatorname{tr}\left[T^{H}(j) T(i)\right]\right\} \\
+\operatorname{Re}\left\{\operatorname { t r } \left[\frac { 1 } { 2 } \left(\sum_{l=1}^{u} A_{l}^{H} R(j+1) B_{l}^{H}\right.\right.\right. \\
+\sum_{s=1}^{v}\left(C_{s}^{H} R(j+1) D_{s}^{H}\right)^{T} \\
+\sum_{l=1}^{u} P A_{l}^{H} R(j+1) B_{l}^{H} Q \\
\left.+\sum_{s=1}^{v} P\left(C_{s}^{H} R(j+1) D_{s}^{H}\right)^{T} Q\right)^{H} \\
\times T(i)]\}
\end{gathered}
$$




$$
\begin{aligned}
= & \frac{\|R(j+1)\|^{2}}{\|R(j)\|^{2}} \operatorname{Re}\left\{\operatorname{tr}\left[T^{H}(j) T(i)\right]\right\} \\
& +\operatorname{Re}\left\{\operatorname { t r } \left[T ^ { H } ( i ) \left(\sum_{l=1}^{u} A_{l}^{H} R(j+1) B_{l}^{H}\right.\right.\right. \\
& \left.\left.\left.+\sum_{s=1}^{v}\left(C_{s}^{H} R(j+1) D_{s}^{H}\right)^{T}\right)\right]\right\} \\
= & \frac{\|R(j+1)\|^{2}}{\|R(j)\|^{2}} \operatorname{Re}\left\{\operatorname{tr}\left[T^{H}(j) T(i)\right]\right\} \\
& +\operatorname{Re}\left\{\operatorname { t r } \left[\sum_{l=1}^{u} R^{H}(j+1) A_{l} T(i) B_{l}\right.\right. \\
= & \frac{\|R(j+1)\|^{2}}{\|R(j)\|^{2}} \operatorname{Re}\left\{\operatorname{tr}\left[T^{H}(j) T(i)\right]\right\} \\
& +\frac{\|T(i)\|^{2}}{\|R(i)\|^{2}}\left(\operatorname{Re}\left\{\operatorname{tr}\left[R^{H}(j+1) R(i)\right]\right\}\right. \\
& +\frac{\|R(j+1)\|^{2}}{\|R(j)\|^{2}} \operatorname{Re}\left\{\operatorname{tr}\left[T^{H}(j) T(i)\right]\right\} \\
& +\operatorname{Re}\left\{\operatorname{tr}\left[R_{s}^{H} R(j+1) D_{s}^{H}\right]\right\} \\
& \frac{\|R(j+1)\|^{2}}{\|R(j)\|^{2}} \operatorname{Re}\left\{\operatorname{tr}\left[T^{H}(j) T(i)\right]\right\} \\
& +\sum_{l=1}^{u} A_{l} T(i) B_{l} \\
& \left.\left.\left.\operatorname{Re} C_{s} T^{T}(i) D_{s}\right)\right]\right\}
\end{aligned}
$$

Therefore, the conclusion (20) holds.

Lemma 8. Assume that the sequences $\{R(i)\}$ and $\{T(i)\}$ are generated by Algorithm 6, and then $\langle R(i), R(j)\rangle=0$ and $\langle T(i), T(j)\rangle=0$ for $i, j=1,2, \ldots, i \neq j$.

Proof. Since $\langle R(i), R(j)\rangle=\langle R(j), R(i)\rangle$ and $\langle T(i), T(j)\rangle=$ $\langle T(j), T(i)\rangle$ for $i, j=1,2, \ldots$, we only need to prove that $\langle R(i), R(j)\rangle=0$ and $\langle T(i), T(j)\rangle=0$ for $1 \leq i<j$.
Now we prove this conclusion by induction. First, we prove

$$
\begin{array}{r}
\langle R(i), R(i+1)\rangle=0, \quad\langle T(i), T(i+1)\rangle=0 \\
\text { for } i=1,2, \ldots
\end{array}
$$

When $i=1$, from Lemma 7 we have

$$
\begin{aligned}
\langle R(1), R(2)\rangle= & \operatorname{Re}\left\{\operatorname{tr}\left[R^{H}(2) R(1)\right]\right\} \\
= & \operatorname{Re}\left\{\operatorname{tr}\left[R^{H}(1) R(1)\right]\right\} \\
& -\frac{\|R(1)\|^{2}}{\|T(1)\|^{2}} \operatorname{Re}\left\{\operatorname{tr}\left[T^{H}(1) S(1)\right]\right\} \\
= & \|R(1)\|^{2}-\frac{\|R(1)\|^{2}}{\|T(1)\|^{2}}\|T(1)\|^{2} \\
= & 0 .
\end{aligned}
$$

From Lemma 7 and conclusion (24), we have

$$
\begin{aligned}
\langle T(1), T(2)\rangle= & \operatorname{Re}\left\{\operatorname{tr}\left[T^{H}(2) T(1)\right]\right\} \\
= & \frac{\|R(2)\|^{2}}{\|R(1)\|^{2}} \operatorname{Re}\left\{\operatorname{tr}\left[T^{H}(1) T(1)\right]\right\} \\
& +\frac{\|T(1)\|^{2}}{\|R(1)\|^{2}}\left(\operatorname{Re}\left\{\operatorname{tr}\left[R^{H}(2) R(1)\right]\right\}\right. \\
= & \frac{\|R(2)\|^{2}}{\|R(1)\|^{2}}\|T(1)\|^{2}-\frac{\|T(1)\|^{2}}{\|R(1)\|^{2}}\|R(2)\|^{2} \\
= & 0 .
\end{aligned}
$$

Now assume that conclusion (23) holds for $1 \leq i \leq t-1$; from Lemma 7, we have

$$
\begin{aligned}
\langle R(t), R(t+1)\rangle \\
=\operatorname{Re}\left\{\operatorname{tr}\left[R^{H}(t+1) R(t)\right]\right\} \\
=\operatorname{Re}\left\{\operatorname{tr}\left[R^{H}(t) R(t)\right]\right\} \\
-\frac{\|R(t)\|^{2}}{\|T(t)\|^{2}} \operatorname{Re}\left\{\operatorname{tr}\left[T^{H}(t) S(t)\right]\right\} \\
=\operatorname{Re}\left\{\operatorname{tr}\left[R^{H}(t) R(t)\right]\right\}-\frac{\|R(t)\|^{2}}{\|T(t)\|^{2}} \\
\quad \times \operatorname{Re}\left\{\operatorname{tr}\left[T^{H}(t)\left(T(t)-\frac{\|R(t)\|^{2}}{\|R(t-1)\|^{2}} T(t-1)\right)\right]\right\} \\
=0 .
\end{aligned}
$$


From Lemma 7 and conclusion (26), it can also be obtained that

$$
\begin{aligned}
& \langle T(t), T(t+1)\rangle \\
& =\operatorname{Re}\left\{\operatorname{tr}\left[T^{H}(t+1) T(t)\right]\right\} \\
& =\frac{\|R(t+1)\|^{2}}{\|R(t)\|^{2}} \operatorname{Re}\left\{\operatorname{tr}\left[T^{H}(t) T(t)\right]\right\} \\
& +\frac{\|T(t)\|^{2}}{\|R(t)\|^{2}}\left(\operatorname{Re}\left\{\operatorname{tr}\left[R^{H}(t+1) R(t)\right]\right\}\right. \\
& =\frac{\|R(t+1)\|^{2}}{\|R(t)\|^{2}}\|T(t)\|^{2}-\frac{\|T(t)\|^{2}}{\|R(t)\|^{2}}\|R(t+1)\|^{2}=0 .
\end{aligned}
$$

Therefore, by the principle of induction, conclusion (23) holds.

Next, assume that $\langle R(i), R(i+r)\rangle=0$ and $\langle T(i), T(i+r)\rangle=$ 0 for $i \geq 1$ and $r \geq 1$. We will prove that

$$
\begin{aligned}
& \langle R(i), R(i+r+1)\rangle=0, \\
& \langle T(i), T(i+r+1)\rangle=0 .
\end{aligned}
$$

In order to prove conclusion (28), we first prove that

$$
\begin{aligned}
& \langle R(1), R(r+2)\rangle=0, \\
& \langle T(1), T(r+2)\rangle=0 .
\end{aligned}
$$

From Lemma 7 we have

$$
\begin{aligned}
& \langle R(1), R(r+2)\rangle \\
& =\operatorname{Re}\left\{\operatorname{tr}\left[R^{H}(r+2) R(1)\right]\right\} \\
& =\operatorname{Re}\left\{\operatorname{tr}\left[R^{H}(r+1) R(1)\right]\right\} \\
& -\frac{\|R(r+1)\|^{2}}{\|T(r+1)\|^{2}} \operatorname{Re}\left\{\operatorname{tr}\left[T^{H}(r+1) S(1)\right]\right\} \\
& =\operatorname{Re}\left\{\operatorname{tr}\left[R^{H}(r+1) R(1)\right]\right\} \\
& -\frac{\|R(r+1)\|^{2}}{\|T(r+1)\|^{2}} \operatorname{Re}\left\{\operatorname{tr}\left[T^{H}(r+1) T(1)\right]\right\} \\
& =0 .
\end{aligned}
$$

From Lemma 7 and conclusion (30), we have

$$
\begin{aligned}
& \langle T(1), T(r+2)\rangle \\
& =\operatorname{Re}\left\{\operatorname{tr}\left[T^{H}(r+2) T(1)\right]\right\} \\
& =\frac{\|R(r+2)\|^{2}}{\|R(r+1)\|^{2}} \operatorname{Re}\left\{\operatorname{tr}\left[T^{H}(r+1) T(1)\right]\right\} \\
& +\frac{\|T(1)\|^{2}}{\|R(1)\|^{2}}\left(\operatorname{Re}\left\{\operatorname{tr}\left[R^{H}(r+2) R(1)\right]\right\}\right. \\
& \left.\quad-\operatorname{Re}\left\{\operatorname{tr}\left[R^{H}(r+2) R(2)\right]\right\}\right) \\
& =0 .
\end{aligned}
$$

Also we have

$$
\begin{aligned}
& \langle R(i), R(i+r+1)\rangle \\
& =\operatorname{Re}\left\{\operatorname{tr}\left[R^{H}(i+r+1) R(i)\right]\right\} \\
& =\operatorname{Re}\left\{\operatorname{tr}\left[R^{H}(i+r) R(i)\right]\right\} \\
& -\frac{\|R(i+r)\|^{2}}{\|T(i+r)\|^{2}} \operatorname{Re}\left\{\operatorname{tr}\left[T^{H}(i+r) S(i)\right]\right\} \\
& =-\frac{\|R(i+r)\|^{2}}{\|T(i+r)\|^{2}} \operatorname{Re}\left\{\operatorname { t r } \left[T^{H}(i+r)\right.\right. \\
& \times\left(T(i)-\frac{\|R(i)\|^{2}}{\|R(i-1)\|^{2}}\right. \\
& \times T(i-1))]\} \\
& =\frac{\|R(i+r)\|^{2}\|R(i)\|^{2}}{\|T(i+r)\|^{2}\|R(i-1)\|^{2}} \\
& \times \operatorname{Re}\left\{\operatorname{tr}\left[T^{H}(i+r) T(i-1)\right]\right\}, \\
& \langle T(i), T(i+r+1)\rangle \\
& =\operatorname{Re}\left\{\operatorname{tr}\left[T^{H}(i+r+1) T(i)\right]\right\} \\
& =\frac{\|R(i+r+1)\|^{2}}{\|R(i+r)\|^{2}} \operatorname{Re}\left\{\operatorname{tr}\left[T^{H}(i+r) T(i)\right]\right\} \\
& +\frac{\|T(i)\|^{2}}{\|R(i)\|^{2}}\left(\operatorname{Re}\left\{\operatorname{tr}\left[R^{H}(i+r+1) R(i)\right]\right\}\right. \\
& -\operatorname{Re}\left\{\operatorname { t r } \left[R^{H}(i+r+1)\right.\right. \\
& \times R(i+1)]\})
\end{aligned}
$$




$$
\begin{aligned}
= & \frac{\|T(i)\|^{2}}{\|R(i)\|^{2}} \operatorname{Re}\left\{\operatorname{tr}\left[R^{H}(i+r+1) R(i)\right]\right\} \\
= & \frac{\|T(i)\|^{2}\|R(i+r)\|^{2}\|R(i)\|^{2}}{\|R(i)\|^{2}\|T(i+r)\|^{2}\|R(i-1)\|^{2}} \\
& \times \operatorname{Re}\left\{\operatorname{tr}\left[T^{H}(i+r) T(i-1)\right]\right\} .
\end{aligned}
$$

Repeating the previous process (32), we have

$$
\begin{aligned}
& \langle R(i), R(i+r+1)\rangle=\cdots=\alpha \operatorname{Re}\left\{\operatorname{tr}\left[T^{H}(r+2) T(1)\right]\right\}, \\
& \langle T(i), T(i+r+1)\rangle=\cdots=\beta \operatorname{Re}\left\{\operatorname{tr}\left[T^{H}(r+2) T(1)\right]\right\} .
\end{aligned}
$$

Combining these two relations with (30) and (31), it implies that (28) holds. So, by the principle of induction, we know that Lemma 8 holds.

Lemma 9. When Problem 1 is consistent, let $\widetilde{X} \in \mathbb{T}_{r}^{n \times n}(P, Q)$ be its solution; then, for any generalized $(P, Q)$-reflexive initial matrix $X(1) \in \mathbb{G}_{r}^{n \times n}(P, Q)$, the sequences $\{R(i)\},\{T(i)\}$ and $\{X(i)\}$ generated by Algorithm 6 satisfy

$$
\langle T(i), \widetilde{X}-X(i)\rangle=\|R(i)\|^{2}, \quad i=1,2, \ldots
$$

Proof. When $i=1$, it follows from Algorithm 6 that

$$
\begin{gathered}
\langle T(1), \widetilde{X}-X(1)\rangle \\
=\operatorname{Re}\left\{\operatorname{tr}\left[(\widetilde{X}-X(1))^{H} T(1)\right]\right\} \\
=\operatorname{Re}\left\{\operatorname { t r } \left[(\widetilde{X}-X(1))^{H}\right.\right. \\
\times\left(\sum_{l=1}^{u} A_{l}^{H} R(1) B_{l}^{H}\right. \\
+\sum_{s=1}^{v}\left(C_{s}^{H} R(1) D_{s}^{H}\right)^{T} \\
+\sum_{l=1}^{u} P A_{l}^{H} R(1) B_{l}^{H} Q \\
\left.\left.\left.+\sum_{s=1}^{v} P\left(C_{s}^{H} R(1) D_{s}^{H}\right)^{T} Q\right) \times 2^{-1}\right]\right\}
\end{gathered}
$$

$$
\begin{aligned}
& =\operatorname{Re}\left\{\operatorname { t r } \left[( \widetilde { X } - X ( 1 ) ) ^ { H } \left(\sum_{l=1}^{u} A_{l}^{H} R(1) B_{l}^{H}\right.\right.\right. \\
& \left.\left.\left.+\sum_{s=1}^{v}\left(C_{s}^{H} R(1) D_{s}^{H}\right)^{T}\right)\right]\right\} \\
& =\operatorname{Re}\left\{\operatorname { t r } \left[R ^ { H } ( 1 ) \left(\sum_{l=1}^{u} A_{l}(\widetilde{X}-X(1)) B_{l}\right.\right.\right. \\
& \left.\left.\left.\quad+\sum_{s=1}^{v} C_{s}\left(\widetilde{X}^{T}-X^{T}(1)\right)\right) D_{s}\right]\right\} \\
& =\operatorname{Re}\left\{\operatorname{tr}\left[R^{H}(1) R(1)\right]\right\} \\
& =\|R(1)\|^{2} .
\end{aligned}
$$

This implies that (34) holds for $i=1$.

Assume that (34) holds for $i=t$, and then, when $i=t+1$,

$$
\begin{gathered}
\langle T(t+1), \widetilde{X}-X(t+1)\rangle \\
=\operatorname{Re}\left\{\operatorname{tr}\left[(\widetilde{X}-X(t+1))^{H} T(t+1)\right]\right\} \\
=\operatorname{Re}\left\{\operatorname { t r } \left[(\widetilde{X}-X(t+1))^{H}\right.\right. \\
\times\left(\frac { 1 } { 2 } \left(\sum_{l=1}^{u} A_{l}^{H} R(t+1) B_{l}^{H}\right.\right. \\
+\sum_{s=1}^{v}\left(C_{s}^{H} R(t+1) D_{s}^{H}\right)^{T} \\
+\sum_{l=1}^{u} P A_{l}^{H} R(t+1) B_{l}^{H} Q \\
\left.+\sum_{s=1}^{v} P\left(C_{s}^{H} R(t+1) D_{s}^{H}\right)^{T} Q\right) \\
\left.\left.\left.+\frac{\|R(t+1)\|^{2}}{\|R(t)\|^{2}} T(t)\right)\right]\right\} \\
+\frac{\operatorname{Re}\left\{\operatorname { t r } \left[(\widetilde{X}-X(t+1))^{H}\right.\right.}{\|R(t)\|^{2}} \operatorname{Re}\left\{\operatorname{tr}\left[(\widetilde{X}-X(t+1))^{H} T(t)\right]\right\} \\
\times\left(\sum_{l=1}^{u} A_{l}^{H} R(t+1) B_{l}^{H}\right. \\
\left.\left.\left.+\sum_{s=1}^{v}\left(C_{s}^{H} R(t+1) D_{s}^{H}\right)^{T}\right)\right]\right\}
\end{gathered}
$$




$$
\begin{aligned}
& \left.\begin{array}{rl}
= & \operatorname{Re} \operatorname{tr}\left[R^{H}(t+1)\right. \\
& \times\left(\sum_{l=1}^{u} A_{l}(\widetilde{X}-X(t+1)) B_{l}\right. \\
& \left.\left.\left.+\sum_{s=1}^{v} C_{s}\left(\widetilde{X}^{T}-X^{T}(t+1)\right) D_{s}\right)\right]\right\} \\
+ & \frac{\|R(t+1)\|^{2}}{\|R(t)\|^{2}} \\
\times & \times\left\{\operatorname{Re}\left\{\operatorname{tr}\left[(\widetilde{X}-X(t))^{H} T(t)\right]\right\}\right. \\
- & \left.\operatorname{Re}\left\{\operatorname{tr}\left[(X(t+1)-X(t))^{H} T(t)\right]\right\}\right\} \\
= & \|R(t+1)\|^{2} \\
+ & \frac{\|R(t+1)\|^{2}}{\|R(t)\|^{2}}\left\{\|R(t)\|^{2}\right. \\
= & \|R(t+1)\|^{2} .
\end{array}-\frac{\|R(t)\|^{2}}{\|T(t)\|^{2}} \operatorname{Re}\left\{\operatorname{tr}\left[T^{H}(t) T(t)\right]\right\}\right\}
\end{aligned}
$$

Therefore, Lemma 9 holds by the principle of induction.

Remark 10. Lemma 9 implies that if there exists a positive integer $i$ such that $R(i) \neq 0$ and $T(i)=0$, then Problem 1 is not consistent. Therefore, the solvability of Problem 1 can be determined by Algorithm 6 in the absence of round-off errors.

From Lemmas 8 and 9, we have the following theorem.

Theorem 11. Assume that Problem 1 is consistent, and then using Algorithm 6 for any generalized $(P, Q)$-reflexive initial matrix $X(1) \in \mathbb{T}_{r}^{n \times n}(P, Q)$, a solution of Problem 1 can be obtained within finite iteration steps in the absence of roundofferrors.

Proof. If $R(i) \neq 0, i=1,2, \ldots, 4 p q$, from Lemma 9 we can obtain $T(i) \neq 0, i=1,2, \ldots, 4 p q$. Thus $R(4 p q+1)$ and $T(4 p q+$ 1) can be computed by Algorithm 6. According to Lemma 8 , we have $\langle R(i), R(j)\rangle=0, i, j=1,2, \ldots, 4 p q$. Considering that the inner product space $\left(\mathbb{M}^{p \times q}, \mathbb{R},\langle\cdot, \cdot\rangle\right)$ is $4 p q$-dimensional, we know that the set of $R(1), R(2), \ldots, R(4 p q)$ is an orthogonal basis of the inner product space $\left(\mathbb{M}^{p \times q}, \mathbb{R},\langle\cdot, \cdot\rangle\right)$. Also from Lemma 8 , we have $\langle R(i), R(4 p q+1)\rangle=0$ for $i=1,2, \ldots, 4 p q$, which implies that $R(4 p q+1)=0$; that is, $X(4 p q+1)$ is a solution of Problem 1.

\section{The Solution of Problem 2}

In this section, we will prove that the sequence $\{X(k)\}$ generated by Algorithm 6 converges to the least Frobenius norm generalized $(P, Q)$-reflexive solution of the quaternion matrix equation $\sum_{l=1}^{u} A_{l} X B_{l}+\sum_{s=1}^{v} C_{s} X^{T} D_{s}=F$ when an appropriate initial iterative matrix is chosen. Then, we solve Problem 2 by finding the least Frobenius norm generalized $(P, Q)$ reflexive solution of a new constructed quaternion matrix equation.

Lemma 12 (see [51]). Assume that the consistent system of linear equations $M y=b$ has a solution $y_{0} \in R\left(M^{T}\right)$; then, $y_{0}$ is the unique least Frobenius norm solution of the system of linear equations.

Lemma 13. If Problem 1 is consistent, then the following system of real linear equations is consistent:

$$
\left[\begin{array}{c}
\left(\begin{array}{c}
\sum_{l=1}^{u} \phi^{T}\left(B_{l}\right) \otimes \phi\left(A_{l}\right)-\sum_{s=1}^{v}\left(\phi^{T}\left(D_{s}\right) \otimes \phi\left(C_{s}\right)\right) P(4 n, 4 n) \\
+2 \sum_{s=1}^{v}\left(\phi^{T}\left(D_{s}\right) \otimes \phi\left(C_{s}\right)\right) \operatorname{diag}(\operatorname{vec}(W)) P(4 n, 4 n)
\end{array}\right) \\
\left(\begin{array}{c}
\sum_{l=1}^{u} \phi^{T}\left(B_{l}\right) \phi(Q) \otimes \phi\left(A_{l}\right) \phi(P) \\
-\sum_{s=1}^{v}\left(\phi^{T}\left(D_{s}\right) \phi(P) \otimes \phi\left(C_{s}\right) \phi(Q)\right) P(4 n, 4 n) \\
+2 \sum_{s=1}^{v}\left(\phi^{T}\left(D_{s}\right) \otimes \phi\left(C_{s}\right)\right) \times \operatorname{diag}(\operatorname{vec}(W))(\phi(P) \otimes \phi(Q)) P(4 n, 4 n)
\end{array}\right)
\end{array}\right] Y=\left[\begin{array}{c}
\operatorname{vec}(\phi(F)) \\
\operatorname{vec}(\phi(F))
\end{array}\right]
$$

Furthermore, if the solution sets of Problem 1 and (37) are denoted by $S_{X}$ and $S_{Y}$, respectively, then,

$$
\operatorname{vec}\left(\phi\left(S_{X}\right)\right) \subseteq S_{Y} .
$$

Proof. If Problem 1 is consistent, let $X$ be a solution of Problem 1, then we have $\sum_{l=1}^{u} A_{l} X B_{l}+\sum_{s=1}^{v} C_{s} X^{T} D_{s}=F$ and $P X Q=X$, which implies that $X$ is a solution of quaternion matrix equations 


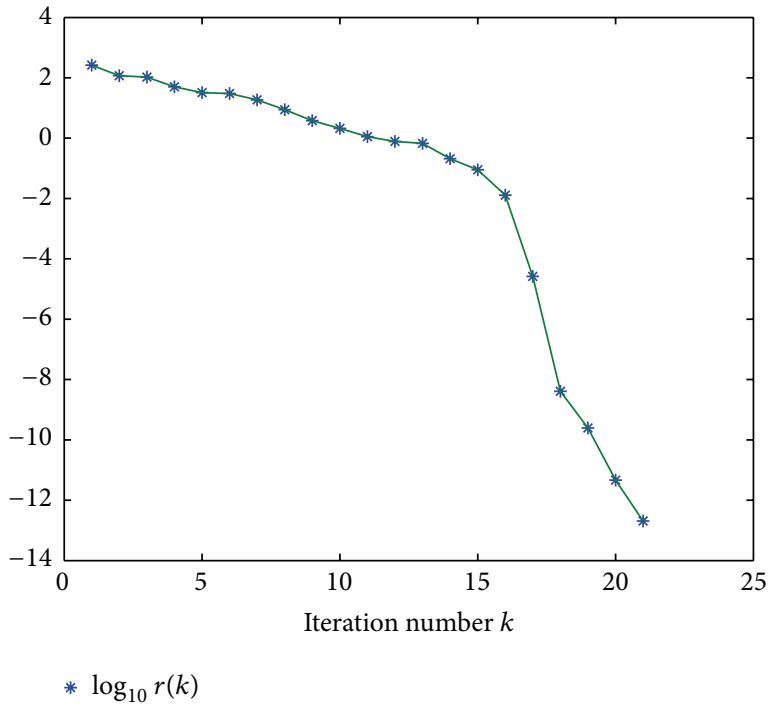

FIGURE 1: The curve for the Frobenius norm of the residuals from Example 17.

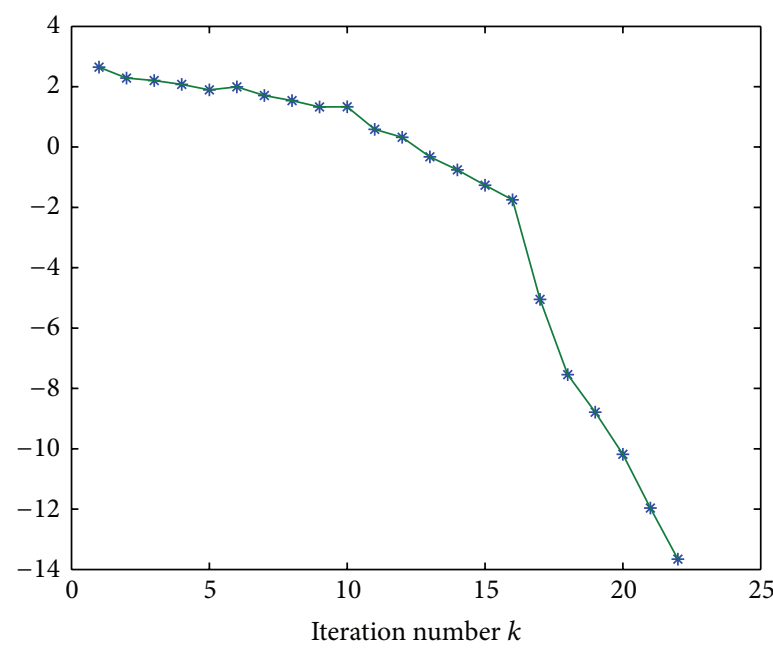

* $\log _{10} r(k)$

Figure 2: The curve for the Frobenius norm of the residuals from Example 18.

$$
\begin{gathered}
\sum_{l=1}^{u} A_{l} X B_{l}+\sum_{s=1}^{v} C_{s} X^{T} D_{s}=F, \\
\sum_{l=1}^{u} A_{l} P X Q B_{l}+\sum_{s=1}^{v} C_{s}(P X Q)^{T} D_{s}=F .
\end{gathered}
$$

By (8), (9), and (12), we can derive that the quaternion matrix equations (39) is equivalent to the following real matrix equations:

$$
\begin{aligned}
\sum_{l=1}^{u} \phi & \left(A_{l}\right) \phi(X) \phi\left(B_{l}\right) \\
& -\sum_{s=1}^{v} \phi\left(C_{s}\right) \phi^{T}(X) \phi\left(D_{s}\right) \\
& +2 \sum_{s=1}^{v} \phi\left(C_{s}\right)\left(\phi^{T}(X) \odot W\right) \phi\left(D_{s}\right)=\phi(F),
\end{aligned}
$$




$$
\begin{aligned}
& \sum_{l=1}^{u} \phi\left(A_{l}\right) \phi(P) \phi(X) \phi(Q) \phi\left(B_{l}\right) \\
& \quad-\sum_{s=1}^{v} \phi\left(C_{s}\right) \phi(Q) \phi^{T}(X) \phi(P) \phi\left(D_{s}\right) \\
& \quad+2 \sum_{s=1}^{v} \phi\left(C_{s}\right)\left(\left(\phi(Q) \phi^{T}(X) \phi(P)\right) \odot W\right) \phi\left(D_{s}\right) \\
& =\phi(F) .
\end{aligned}
$$

By the operation properties of vec $(\cdot), \otimes, \odot$, and Lemma 4 , it is not difficult to verify that matrix equations (40) is equivalent to

$$
\left[\begin{array}{c}
\left(\begin{array}{c}
\sum_{l=1}^{u} \phi^{T}\left(B_{l}\right) \otimes \phi\left(A_{l}\right)-\sum_{s=1}^{v}\left(\phi^{T}\left(D_{s}\right) \otimes \phi\left(C_{s}\right)\right) P(4 n, 4 n) \\
+2 \sum_{s=1}^{v}\left(\phi^{T}\left(D_{s}\right) \otimes \phi\left(C_{s}\right)\right) \operatorname{diag}(\operatorname{vec}(W)) P(4 n, 4 n)
\end{array}\right) \\
\left(\begin{array}{c}
\sum_{l=1}^{u} \phi^{T}\left(B_{l}\right) \phi(Q) \otimes \phi\left(A_{l}\right) \phi(P) \\
-\sum_{s=1}^{v}\left(\phi^{T}\left(D_{s}\right) \phi(P) \otimes \phi\left(C_{s}\right) \phi(Q)\right) P(4 n, 4 n) \\
+2 \sum_{s=1}^{v}\left(\phi^{T}\left(D_{s}\right) \otimes \phi\left(C_{s}\right)\right) \times \operatorname{diag}(\operatorname{vec}(W))(\phi(P) \otimes \phi(Q)) P(4 n, 4 n)
\end{array}\right) \\
\times \operatorname{vec}(\phi(X))=\left[\begin{array}{c}
\operatorname{vec}(\phi(F)) \\
\operatorname{vec}(\phi(F))
\end{array}\right]
\end{array}\right]
$$

which implies that $\operatorname{vec}(\phi(X))$ is a solution of (37). So system of real linear equations (37) is consistent.

From the previous procedure, the proof of (38) is trivial.

Theorem 14. If $\stackrel{\circ}{X}$ is a solution of Problem 1 and $\stackrel{\circ}{X}$ can be expressed as the following form:

$$
\stackrel{\circ}{X}=\sum_{l=1}^{u} A_{l}^{H} G B_{l}^{H}+\sum_{s=1}^{v}\left(C_{s}^{H} G D_{s}^{H}\right)^{T}
$$

$$
\begin{aligned}
& +\sum_{l=1}^{u} P A_{l}^{H} G B_{l}^{H} Q \\
& +\sum_{s=1}^{v} P\left(C_{s}^{H} G D_{s}^{H}\right)^{T} Q, \quad G \in \mathbb{H}^{p \times q}
\end{aligned}
$$

then, $\stackrel{\circ}{X}$ is the least Frobenius norm solution of Problem 1.

Proof. By (8), (9), (10), and (12) and Lemmas 4 and 5, we have

$$
\begin{aligned}
& \operatorname{vec}(\phi(\dot{X})) \\
& =\operatorname{vec}\left(\sum_{l=1}^{u} \phi^{T}\left(A_{l}\right) \phi(G) \phi^{T}\left(B_{l}\right)+2 \sum_{s=1}^{v}\left(\phi\left(D_{s}\right) \phi^{T}(G) \phi\left(C_{s}\right)\right) \odot W\right. \\
& \quad-\sum_{s=1}^{v} \phi\left(D_{s}\right) \phi^{T}(G) \phi\left(C_{s}\right)+\sum_{l=1}^{u} \phi(P) \phi^{T}\left(A_{l}\right) \phi(G) \phi^{T}\left(B_{l}\right) \phi(Q) \\
& \left.\quad+2 \sum_{s=1}^{v} \phi(P)\left(\left(\phi\left(D_{s}\right) \phi^{T}(G) \phi\left(C_{s}\right)\right) \odot W\right) \phi(Q)-\sum_{s=1}^{v} \phi(P) \phi\left(D_{s}\right) \phi^{T}(G) \phi\left(C_{s}\right) \phi(Q)\right)
\end{aligned}
$$




$$
\begin{aligned}
& =\left[\sum_{l=1}^{u} \phi\left(B_{l}\right) \otimes \phi^{T}\left(A_{l}\right)+2 \sum_{s=1}^{v} \operatorname{diag}(\operatorname{vec}(W))\left(\phi^{T}\left(C_{s}\right) \otimes \phi\left(D_{s}\right)\right) P(4 p, 4 q)\right. \\
& -\sum_{s=1}^{v}\left(\phi^{T}\left(C_{s}\right) \otimes \phi\left(D_{s}\right)\right) P(4 p, 4 q), \\
& \sum_{l=1}^{u} \phi(Q) \phi\left(B_{l}\right) \otimes \phi(P) \phi^{T}\left(A_{l}\right)+2 \sum_{s=1}^{v}(\phi(Q) \otimes \phi(P)) \operatorname{diag}(\operatorname{vec}(W))\left(\phi^{T}\left(C_{s}\right) \otimes \phi\left(D_{s}\right)\right) P(4 p, 4 q) \\
& \left.-\sum_{s=1}^{v}\left(\phi(Q) \phi^{T}\left(C_{s}\right) \otimes \phi(P) \phi\left(D_{s}\right)\right) P(4 p, 4 q)\right]\left[\begin{array}{l}
\operatorname{vec}(\phi(G)) \\
\operatorname{vec}(\phi(G))
\end{array}\right]
\end{aligned}
$$

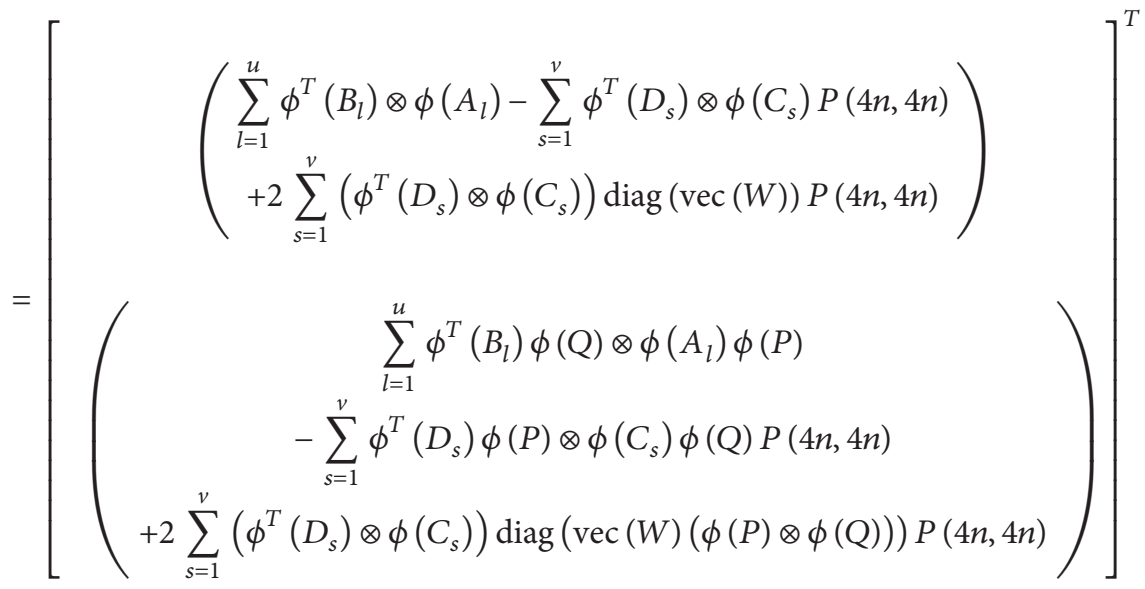

$$
\begin{aligned}
& \times\left[\begin{array}{c}
\operatorname{vec}(\phi(G)) \\
\operatorname{vec}(\phi(G))
\end{array}\right]
\end{aligned}
$$

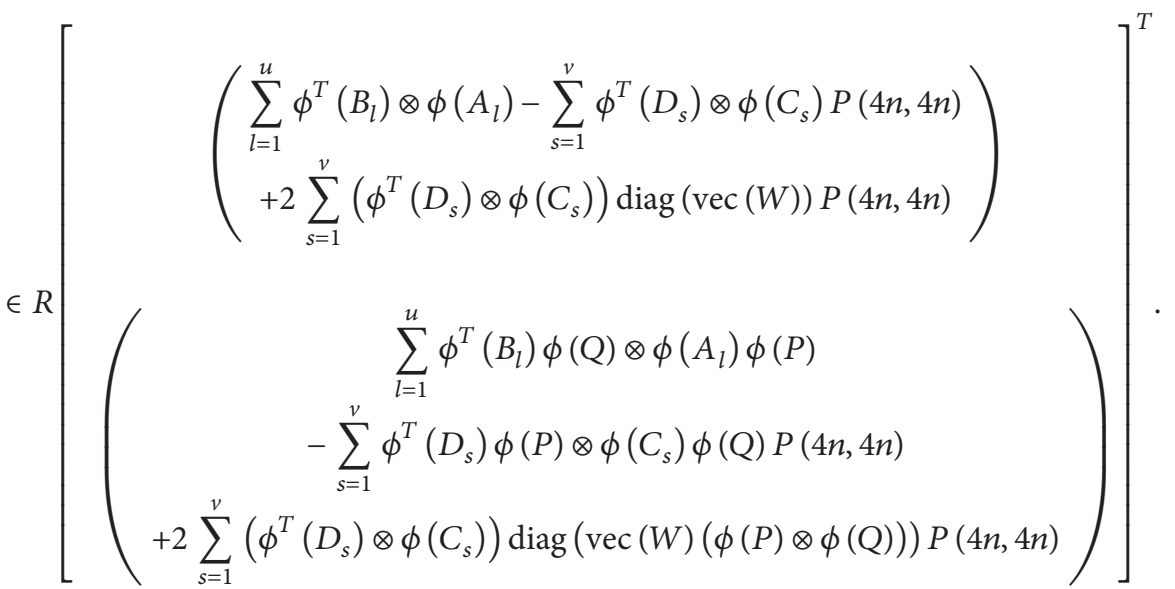

By Lemma 12, $\operatorname{vec}(\phi(\stackrel{X}{X}))$ is the least Frobenius norm solution of (37).

Noting (11), we derive from Lemma 13 that ${ }_{X}$ is the least Frobenius norm solution of Problem 1.

Remark 15. From Algorithm 6, we can derive that if we choose $X(1)=\sum_{l=1}^{u} A_{l}^{H} G B_{l}^{H}+\sum_{s=1}^{v}\left(C_{s}^{H} G D_{s}^{H}\right)^{T}+$ $\sum_{l=1}^{u} P A_{l}^{H} G B_{l}^{H} Q+\sum_{s=1}^{v} P\left(C_{s}^{H} G D_{s}^{H}\right)^{T} Q, G \in \mathbb{\mathbb { A } ^ { p \times q }}$, as the initial iteration matrix, then all $X(k)$ generated by Algorithm 6 have a form of

$$
\begin{aligned}
X(k)= & \sum_{l=1}^{u} A_{l}^{H} G_{k} B_{l}^{H}+\sum_{s=1}^{v}\left(C_{s}^{H} G_{k} D_{s}^{H}\right)^{T} \\
& +\sum_{l=1}^{u} P A_{l}^{H} G_{k} B_{l}^{H} Q \\
& +\sum_{s=1}^{v} P\left(C_{s}^{H} G_{k} D_{s}^{H}\right)^{T} Q, \quad G_{k} \in \mathbb{\mathbb { T } ^ { p \times q } .}
\end{aligned}
$$


Theorem 16. When Problem 1 is consistent, if we choose the following matrix as the initial iteration matrix

$$
\begin{aligned}
X(1)= & \sum_{l=1}^{u} A_{l}^{H} G B_{l}^{H}+\sum_{s=1}^{v}\left(C_{s}^{H} G D_{s}^{H}\right)^{T} \\
& +\sum_{l=1}^{u} P A_{l}^{H} G B_{l}^{H} Q+\sum_{s=1}^{v} P\left(C_{s}^{H} G D_{s}^{H}\right)^{T} Q,
\end{aligned}
$$

where $G \in \mathbb{M}^{p \times q}$ is arbitrary, or especially, $X(1)=0$, then the solution $X^{*}$, generated by Algorithm 6, is exactly the least Frobenius norm solution of Problem 1.

From conclusion (44) and Theorem 14, the proof of Theorem 16 is trivial.

Now we consider Problem 2. When Problem 1 is consistent, the solution set of Problem $1 S_{X}$ is not empty. For a given generalized $(P, Q)$-reflexive matrix $X_{0} \in \mathbb{T}_{r}^{n \times n}(P, Q)$, let $\dot{X}=$ $X-X_{0}, \dot{F}=F-\sum_{l=1}^{u} A_{l} X_{0} B_{l}-\sum_{s=1}^{v} C_{s} X_{0}^{T} D_{s}$; then the least Frobenius norm generalized $(P, Q)$-reflexive solution of the following quaternion matrix equation is exactly the solution of $\min _{X \in S_{X}}\left\|X-X_{0}\right\|$

$$
\sum_{l=1}^{u} A_{l} \dot{X} B_{l}+\sum_{s=1}^{v} C_{s} \dot{X}^{T} D_{s}=\dot{F} .
$$

According to Theorem 16, if we choose the initial iteration matrix $\dot{X}(1)=\sum_{l=1}^{u} A_{l}^{H} G B_{l}^{H}+\sum_{s=1}^{v}\left(C_{s}^{H} G D_{s}^{H}\right)^{T}+$ $\sum_{l=1}^{u} P A_{l}^{H} G B_{l}^{H} Q+\sum_{s=1}^{v} P\left(C_{s}^{H} G D_{s}^{H}\right)^{T} Q$, where $G \in \mathbb{H}^{p \times q}$ is arbitrary, or especially, choosing $\dot{X}(1)=0$, the least Frobenius norm generalized $(P, Q)$-reflexive solution $\dot{X}^{*}$ of (46) can be obtained. Then the solution of Problem 2 is $\widehat{X}=$ $\dot{X}^{*}+X_{0}$.

\section{Examples}

In this section, we give two examples to illustrate the performance of the proposed algorithm.

Example 17. Consider the quaternion matrix equation

$$
A_{1} X B_{1}+C_{1} X^{T} D_{1}+A_{2} X B_{2}+C_{2} X^{T} D_{2}=F
$$

with the matrices

$$
\begin{aligned}
& A_{1}=\left[\begin{array}{cccc}
2+i+2 j & 1+2 i+2 j+k & 3 j & 2 i+j+2 k \\
2 i+2 j+k & -1+3 k & 1+2 i+4 j+2 k & 4+i+2 j+4 k
\end{array}\right], \\
& A_{2}=\left[\begin{array}{cccc}
i+j+k & i & 1-2 i+j+2 k & 2+4 i+2 k \\
2 i+2 j+k & 1+3 i+j & 3+4 i+j-2 k & 2+2 i+2 j+k
\end{array}\right], \\
& B_{1}=\left[\begin{array}{cc}
1+5 i+2 j+k & 2+3 i+3 j+2 k \\
6 i+4 j-k & 2+5 j \\
2+2 i+j-k & 4+7 i+2 j+2 k \\
3+j-k & 1+2 i-2 j+3 k
\end{array}\right] \\
& B_{2}=\left[\begin{array}{cc}
-i+4 j+k & -3+2 i+3 j+2 k \\
2+4 i-k & -1+5 i+2 j-2 k \\
1+6 i+4 j+5 k & 2-3 i+j+k \\
-i+6 j+3 k & 1+7 i-2 j-4 k
\end{array}\right] \\
& C_{1}=\left[\begin{array}{cccc}
i+2 j+k & 1+i-3 j+2 k & 1+5 i+k & 2 i+4 j-4 k \\
1-4 i+2 j+k & 5+5 i-j+k & -4+2 i+3 j+2 k & 1+5 i+2 j-4 k
\end{array}\right], \\
& C_{2}=\left[\begin{array}{cccc}
1+i+3 j & 3-i+3 j+k & 1+i+5 j+3 k & 2+4 i+2 j \\
5+3 i+j+4 k & 2+i+2 j+3 k & 3+4 i+3 j+2 k & -2+i-3 j+k
\end{array}\right], \\
& D_{1}=\left[\begin{array}{cc}
2+6 i+6 j+k & 1+3 i+j-2 k \\
-4 i+3 j+8 k & -2-2 i-4 j+5 k \\
5+3 i-2 j+4 k & 1+2 i+j+2 k \\
3-i+2 j-2 k & 3+i-j+3 k
\end{array}\right], \quad D_{2}=\left[\begin{array}{cc}
i-5 j+6 k & -2-8 i-4 j-3 k \\
3+4 i+7 j+2 k & 5 i+2 j \\
5+7 i-2 j-k & -2+9 i+7 j+k \\
1+3 i+4 j & 6+2 j+7 k
\end{array}\right] \text {, } \\
& F=\left[\begin{array}{cc}
6+i+2 j+k & -8-7 i-3 j+2 k \\
i-6 j-4 k & 3-2 i+6 k
\end{array}\right]
\end{aligned}
$$


We apply Algorithm 6 to find the generalized $(P, Q)$-reflexive solution of (47), where

$$
\begin{gathered}
P=\left[\begin{array}{cccc}
0.28 & 0 & 0.96 k & 0 \\
0 & 0.28 & 0 & -0.96 j \\
-0.96 k & 0 & -0.28 & 0 \\
0 & 0.96 j & 0 & -0.28
\end{array}\right] \\
Q=\left[\begin{array}{cccc}
0.28 & 0 & 0.96 k & 0 \\
0 & -1 & 0 & 0 \\
-0.96 k & 0 & -0.28 & 0 \\
0 & 0 & 0 & -1
\end{array}\right]
\end{gathered}
$$

$$
\begin{aligned}
& X^{*}=X(21) \\
& =\left[\begin{array}{c}
0.51783+0.066965 i+0.095973 j-0.025639 k \\
-0.13818-0.050488 i-0.073437 j+0.11425 k \\
0.026384+0.045766 i-0.22964 j+0.066304 k \\
0.12175+0.14209 i-0.11665 j+0.20690 k
\end{array}\right. \\
& -0.026384+0.045766 i-0.22964 j-0.066304 k \\
& -0.14209-0.12175 i+0.20690 j-0.11665 k \\
& 0.55651+0.066993 i-0.069276 j-0.041030 k \\
& 0.070202-0.070130 i-0.031364 j+0.0024134 k
\end{aligned}
$$

are two generalized reflection matrices. Choosing an initial matrix

$$
X(1)=\left[\begin{array}{cccc}
1 & 0 & 0 & 0 \\
0 & 0.36 & 0 & 0.48 j \\
0 & 0 & 1 & 0 \\
0 & -0.48 j & 0 & 0.64
\end{array}\right] \in \mathbb{H}_{r}^{4 \times 4}(P, Q)
$$

we obtain that the sequence $\{X(k)\}$ after 21 steps is with $\|R(21)\|=2.047 \times 10^{-13}$. The obtained results are presented in Figure 1, where $r(k)=\|R(k)\|$.

\section{Example 18. Let}

$$
X_{0}=\left[\begin{array}{cccc}
0.64 & -0.72 i-0.75 k & 0.48 k & -0.5625 i-0.96 k \\
0.64+0.36 i & 0 & 0.48 j+0.48 k & 0 \\
-0.48 k & 1-0.96 j & 0.36 & 1.28-0.75 j \\
0.48 j+0.48 k & 0 & 0.64+0.36 i & 0
\end{array}\right] \in \mathbb{M}_{r}^{4 \times 4}(P, Q)
$$

Now we consider Problem 2, that is, finding the optimal approximate generalized $(P, Q)$-reflexive solution of the quaternion matrix equation (47) to $X_{0}$. Applying Algorithm 6 to (46) by choosing the initial matrix $\dot{X}(1)=0$, we obtain that the sequence $\{X(k)\}$ after 22 steps is

$$
\begin{aligned}
& \dot{X}^{*}=\dot{X}(22) \\
& =\left[\begin{array}{ccc}
-0.60797+0.14851 i-0.031273 j+0.032158 k & 0.093923+0.086087 i+0.24350 j+0.44225 k \\
-0.32942-0.34127 i-0.091587 j+0.36047 k & -0.092590-0.15547 i-0.19614 j+0.067121 k \\
-0.087200-0.13538 i+0.090255 j+0.25660 k & -0.58966-0.32467 i+0.11478 j+0.12523 k \\
0.020667+0.14412 i-0.17405 j+0.058232 k & -0.26152-0.089494 i+0.12345 j-0.20729 k
\end{array}\right. \\
& 0.087200-0.13538 i+0.090255 j-0.25660 k-0.021779+0.31853 i-0.55293 j+0.58717 k \\
& -0.14412-0.020667 i+0.058232 j-0.17405 k \quad 0.090225-0.057613 i-0.17618 j-0.066567 k \\
& -0.45828-0.20116 i-0.047696 j+0.083025 k-0.78290+0.73724 i+0.42471 j-0.029038 k \text {, }
\end{aligned}
$$

with $\|\dot{R}(22)\|=2.1855 \times 10^{-14}$. The obtained results are presented in Figure 2, where $r(k)=\|\dot{R}(k)\|$.
So we can obtain the solution of Problem 2; that is, 


$$
\begin{aligned}
& \widehat{X}=\dot{X}^{*}+X_{0} \\
& =\left[\begin{array}{cc}
0.032031+0.14851 i-0.031273 j+0.032158 k & 0.093923-0.63391 i+0.24350 j-0.30775 k \\
0.31058+0.018728 i-0.091587 j+0.36047 k & -0.092590-0.15547 i-0.19614 j+0.067121 k \\
-0.087200-0.13538 i+0.090255 j-0.22340 k & 0.41034-0.32467 i-0.84522 j+0.12523 k \\
0.020667+0.14412 i+0.30595 j+0.53823 k & -0.26152-0.089494 i+0.12345 j-0.20729 k
\end{array}\right. \\
& 0.087200-0.13538 i+0.090255 j+0.22340 k \quad-0.021779-0.24397 i-0.55293 j-0.37283 k \\
& -0.14412-0.020667 i+0.53823 j+0.30595 k \quad 0.090225-0.057613 i-0.17618 j-0.066567 k \\
& -0.098285-0.20116 i-0.047696 j+0.083025 k \\
& 0.33270+0.13211 i-0.27640 j+0.079531 k \\
& 0.49710+0.73724 i-0.32529 j-0.029038 k \\
& -0.23490+0.088756 i-0.12030 j-0.076818 k
\end{aligned}
$$

\section{Conclusions}

In this paper, an algorithm has been presented for solving the quaternion matrix equation $\sum_{l=1}^{u} A_{l} X B_{l}+\sum_{s=1}^{v} C_{s} X^{T} D_{s}=F$ over generalized $(P, Q)$-reflexive matrices. It has been proven that when the quaternion matrix equation $\sum_{l=1}^{u} A_{l} X B_{l}+$ $\sum_{s=1}^{v} C_{s} X^{T} D_{s}=F$ is consistent over generalized $(P, Q)$ reflexive matrices, for any generalized $(P, Q)$-reflexive initial iterative matrix, the sequence $\{X(k)\}$ generated by Algorithm 6 will converge to a generalized $(P, Q)$-reflexive solution within finite iteration steps in the absence of roundoff errors. We have also proven that by choosing a suitable initial iterative matrix, the sequence $\{X(k)\}$ will converge to the least Frobenius norm generalized $(P, Q)$-reflexive solution of the quaternion matrix equation $\sum_{l=1}^{u} A_{l} X B_{l}+\sum_{s=1}^{v} C_{s} X^{T} D_{s}=$ $F$. Then, by using Algorithm 6, we solved Problem 2. The numerical results have shown that the presented algorithm is quite effective.

\section{Acknowledgments}

This research was supported by the grants from the Innovation Program of Shanghai Municipal Education Commission (13ZZ080), the National Natural Science Foundation of China (11171205), and the Natural Science Foundation of Shanghai (11ZR1412500).

\section{References}

[1] I. Kyrchei, "Explicit representation formulas for the minimum norm least squares solutions of some quaternion matrix equations," Linear Algebra and its Applications, vol. 438, no. 1, pp. 136-152, 2013.

[2] S. Yuan and A. Liao, "Least squares solution of the quaternion matrix equation $X-A \widehat{X} B=C$ with the least norm," Linear and Multilinear Algebra, vol. 59, no. 9, pp. 985-998, 2011.

[3] C. Song, G. Chen, and X. Wang, "On solutions of quaternion matrix equations $X F-A X=B Y$ and $X F-A \widetilde{X}=B Y$," Acta Mathematica Scientia B, vol. 32, no. 5, pp. 1967-1982, 2012.

[4] Z.-H. He and Q.-W. Wang, "A real quaternion matrix equation with applications," Linear and Multilinear Algebra, vol. 61, no. 6, pp. 725-740, 2013.

[5] H.-C. Chen and A. H. Sameh, "A matrix decomposition method for orthotropic elasticity problems," SIAM Journal on Matrix Analysis and Applications, vol. 10, no. 1, pp. 39-64, 1989.
[6] H.-C. Chen, "Generalized reflexive matrices: special properties and applications," SIAM Journal on Matrix Analysis and Applications, vol. 19, no. 1, pp. 140-153, 1998.

[7] L. Datta and S. D. Morgera, "On the reducibility of centrosymmetric matrices-applications in engineering problems," Circuits, Systems, and Signal Processing, vol. 8, no. 1, pp. 71-96, 1989.

[8] Z.-h. Peng, X.-y. Hu, and L. Zhang, "An efficient algorithm for the least-squares reflexive solution of the matrix equation $A_{1} X B_{1}=C_{1}, A_{2} X B_{2}=C_{2}$," Applied Mathematics and Computation, vol. 181, no. 2, pp. 988-999, 2006.

[9] Y.-X. Peng, X.-Y. Hu, and L. Zhang, "An iterative method for symmetric solutions and optimal approximation solution of the system of matrix equations $A_{1} X B_{1}=C_{1}, A_{2} X B_{2}=C_{2}$," Applied Mathematics and Computation, vol. 183, no. 2, pp. 1127-1137, 2006.

[10] F. Ding and T. Chen, "Gradient based iterative algorithms for solving a class of matrix equations," IEEE Transactions on Automatic Control, vol. 50, no. 8, pp. 1216-1221, 2005.

[11] F. Ding and T. Chen, "On iterative solutions of general coupled matrix equations," SIAM Journal on Control and Optimization, vol. 44, no. 6, pp. 2269-2284, 2006.

[12] F. Ding and T. Chen, "Hierarchical gradient-based identification of multivariable discrete-time systems," Automatica, vol. 41, no. 2, pp. 315-325, 2005.

[13] Z.-Y. Peng, "A matrix LSQR iterative method to solve matrix equation $A X B=C$," International Journal of Computer Mathematics, vol. 87, no. 8, pp. 1820-1830, 2010.

[14] Z.-y. Peng, "New matrix iterative methods for constraint solutions of the matrix equation $A X B=C$," Journal of Computational and Applied Mathematics, vol. 235, no. 3, pp. 726-735, 2010.

[15] Z.-Y. Peng, "Solutions of symmetry-constrained least-squares problems," Numerical Linear Algebra with Applications, vol. 15, no. 4, pp. 373-389, 2008.

[16] C. C. Paige, "Bidiagonalization of matrices and solutions of the linear equations," SIAM Journal on Numerical Analysis, vol. 11, pp. 197-209, 1974.

[17] M. Wang, X. Cheng, and M. Wei, "Iterative algorithms for solving the matrix equation $A X B+C X^{T} D=E$, Applied Mathematics and Computation, vol. 187, no. 2, pp. 622-629, 2007.

[18] L. Xie, J. Ding, and F. Ding, "Gradient based iterative solutions for general linear matrix equations," Computers \& Mathematics with Applications, vol. 58, no. 7, pp. 1441-1448, 2009.

[19] Z.-Y. Li, Y. Wang, B. Zhou, and G.-R. Duan, "Least squares solution with the minimum-norm to general matrix equations via iteration," Applied Mathematics and Computation, vol. 215, no. 10 , pp. 3547-3562, 2010. 
[20] X. Duan, A. Liao, and B. Tang, "On the nonlinear matrix equation $X-\sum_{i=1}^{m} A_{i}^{*} X^{\delta_{i}} A_{i}=$ Q," Linear Algebra and its Applications, vol. 429, no. 1, pp. 110-121, 2008.

[21] X. Duan and A. Liao, "On the nonlinear matrix equation $X+$ $A^{*} X^{-q} A=Q(q \geq 1)$," Mathematical and Computer Modelling, vol. 49, no. 5-6, pp. 936-945, 2009.

[22] X. Duan and A. Liao, "On Hermitian positive definite solution of the matrix equation $X-\sum_{i=1}^{m} A_{i}^{*} X^{r} A_{i}=Q$," Journal of Computational and Applied Mathematics, vol. 229, no. 1, pp. 27-36, 2009.

[23] X. Duan, C. Li, and A. Liao, "Solutions and perturbation analsis for the nonlinear matrix equation $X+\sum_{i=1}^{m} A_{i}^{*} X^{-1} A_{i}=I$," Applied Mathematics and Computation, vol. 218, no. 8, pp. 44584466, 2011.

[24] X. Duan and A. Liao, "On the existence of Hermitian positive definite solutions of the matrix equation $X^{s}+A^{*} X^{-t} A=Q$," Linear Algebra and its Applications, vol. 429, no. 4, pp. 673-687, 2008.

[25] M. Dehghan and M. Hajarian, "Finite iterative algorithms for the reflexive and anti-reflexive solutions of the matrix equation $A_{1} X_{1} B_{1}+A_{2} X_{2} B_{2}=C$," Mathematical and Computer Modelling, vol. 49, no. 9-10, pp. 1937-1959, 2009.

[26] M. Dehghan and M. Hajarian, "Two iterative algorithms for solving coupled matrix equations over reflexive and anti-reflexive matrices," Computational \& Applied Mathematics, vol. 31, no. 2, pp. 353-371, 2012.

[27] M. Dehghan and M. Hajarian, "Analysis of an iterative algorithm to solve the generalized coupled Sylvester matrix equations," Applied Mathematical Modelling, vol. 35, no. 7, pp. 32853300, 2011.

[28] M. Hajarian and M. Dehghan, "The generalized centro-symmetric and least squares generalized centro-symmetric solutions of the matrix equation $A Y B+C Y^{T} D=E$, Mathematical Methods in the Applied Sciences, vol. 34, no. 13, pp. 1562-1579, 2011.

[29] M. Dehghan and M. Hajarian, "The general coupled matrix equations over generalized bisymmetric matrices," Linear Algebra and its Applications, vol. 432, no. 6, pp. 1531-1552, 2010.

[30] M. Dehghan and M. Hajarian, "An iterative method for solving the generalized coupled Sylvester matrix equations over generalized bisymmetric matrices," Applied Mathematical Modelling. Simulation and Computation for Engineering and Environmental Systems, vol. 34, no. 3, pp. 639-654, 2010.

[31] A.-G. Wu, X. Zeng, G.-R. Duan, and W.-J. Wu, "Iterative solutions to the extended Sylvester-conjugate matrix equations," Applied Mathematics and Computation, vol. 217, no. 1, pp. 130$142,2010$.

[32] A.-G. Wu, G. Feng, G.-R. Duan, and W.-J. Wu, "Iterative solutions to coupled Sylvester-conjugate matrix equations," Computers \& Mathematics with Applications, vol. 60, no. 1, pp. 54-66, 2010.

[33] A.-G. Wu, B. Li, Y. Zhang, and G.-R. Duan, "Finite iterative solutions to coupled Sylvester-conjugate matrix equations," Applied Mathematical Modelling, vol. 35, no. 3, pp. 1065-1080, 2011.

[34] A.-G. Wu, L. Lv, and M.-Z. Hou, "Finite iterative algorithms for a common solution to a group of complex matrix equations," Applied Mathematics and Computation, vol. 218, no. 4, pp. 11911202, 2011.

[35] A.-G. Wu, L. Lv, and M.-Z. Hou, "Finite iterative algorithms for extended Sylvester-conjugate matrix equations," Mathematical and Computer Modelling, vol. 54, no. 9-10, pp. 2363-2384, 2011.

[36] M. Wang, M. Wei, and Y. Feng, "An iterative algorithm for least squares problem in quaternionic quantum theory," Computer Physics Communications, vol. 179, no. 4, pp. 203-207, 2008.
[37] F. Zhang, "Quaternions and matrices of quaternions," Linear Algebra and its Applications, vol. 251, pp. 21-57, 1997.

[38] N. Le Bihan and J. Mars, "Singular value decomposition of quaternion matrices: a new tool for vector-sensor signal processing," Signal Processing, vol. 84, no. 7, pp. 1177-1199, 2004.

[39] F. O. Farid, Q.-W. Wang, and F. Zhang, "On the eigenvalues of quaternion matrices," Linear and Multilinear Algebra, vol. 59, no. 4, pp. 451-473, 2011.

[40] C. C. Took, D. P. Mandic, and F. Zhang, "On the unitary diagonalisation of a special class of quaternion matrices," Applied Mathematics Letters, vol. 24, no. 11, pp. 1806-1809, 2011.

[41] S. J. Sangwine and N. Le Bihan, "Quaternion singular value decomposition based on bidiagonalization to a real or complex matrix using quaternion Householder transformations," Applied Mathematics and Computation, vol. 182, no. 1, pp. 727-738, 2006.

[42] S. De Leo and G. Scolarici, "Right eigenvalue equation in quaternionic quantum mechanics," Journal of Physics A, vol. 33, no. 15, pp. 2971-2995, 2000.

[43] Q.-W. Wang, X. Liu, and S.-W. Yu, "The common bisymmetric nonnegative definite solutions with extreme ranks and inertias to a pair of matrix equations," Applied Mathematics and Computation, vol. 218, no. 6, pp. 2761-2771, 2011.

[44] Q.-W. Wang, Y. Zhou, and Q. Zhang, "Ranks of the common solution to six quaternion matrix equations," Acta Mathematicae Applicatae Sinica, vol. 27, no. 3, pp. 443-462, 2011.

[45] Q.-W. Wang, H.-X. Chang, and C.-Y. Lin, "P-(skew)symmetric common solutions to a pair of quaternion matrix equations," Applied Mathematics and Computation, vol. 195, no. 2, pp. 721732, 2008.

[46] Q.-W. Wang, J. W. van der Woude, and H.-X. Chang, "A system of real quaternion matrix equations with applications," Linear Algebra and its Applications, vol. 431, no. 12, pp. 2291-2303, 2009.

[47] Q. Wang, S. Yu, and W. Xie, "Extreme ranks of real matrices in solution of the quaternion matrix equation $A X B=C$ with applications," Algebra Colloquium, vol. 17, no. 2, pp. 345-360, 2010.

[48] L. R. Fletcher, J. Kautsky, and N. K. Nichols, "Eigenstructure assignment in descriptor systems," IEEE Transactions on Automatic Control, vol. AC-31, no. 12, pp. 1138-1141, 1986.

[49] L. Dai, Singular Control Systems, vol. 118 of Lecture Notes in Control and Information Sciences, Springer, Berlin, Germany, 1989.

[50] P. M. Frank, "Fault diagnosis in dynamic systems using analytical and knowledge-based redundancy: a survey and some new results," Automatica, vol. 26, no. 3, pp. 459-474, 1990.

[51] Y.-X. Peng, X.-Y. Hu, and L. Zhang, "An iteration method for the symmetric solutions and the optimal approximation solution of the matrix equation $A X B=C$," Applied Mathematics and Computation, vol. 160, no. 3, pp. 763-777, 2005.

[52] L. Xie, Y. Liu, and H. Yang, "Gradient based and least squares based iterative algorithms for matrix equations $A X B+C X^{T} D=$ F," Applied Mathematics and Computation, vol. 217, no. 5, pp. 2191-2199, 2010.

[53] F. Piao, Q. Zhang, and Z. Wang, "The solution to matrix equation $A X+X^{T} C=B$," Journal of the Franklin Institute, vol. 344, no. 8, pp. 1056-1062, 2007.

[54] X. D. Zhang, Matrix Analysis and Applications, Tsinghua University Press, Beijing, China, 2004.

[55] R. A. Horn and C. R. Johnson, Topics in Matrix Analysis, Cambridge University Press, Cambridge, UK, 1991. 


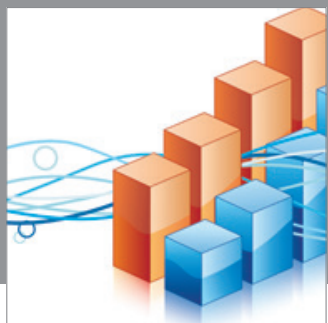

Advances in

Operations Research

mansans

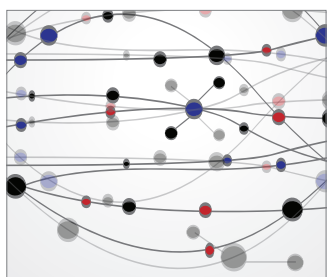

The Scientific World Journal
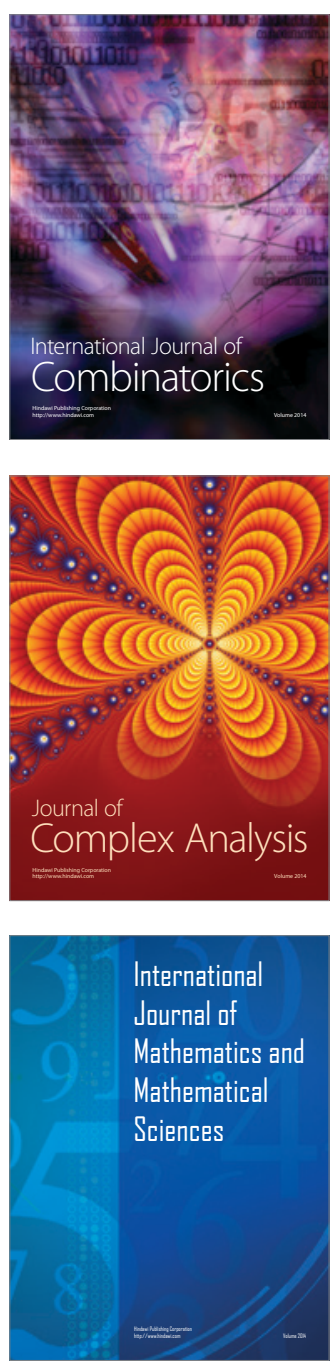
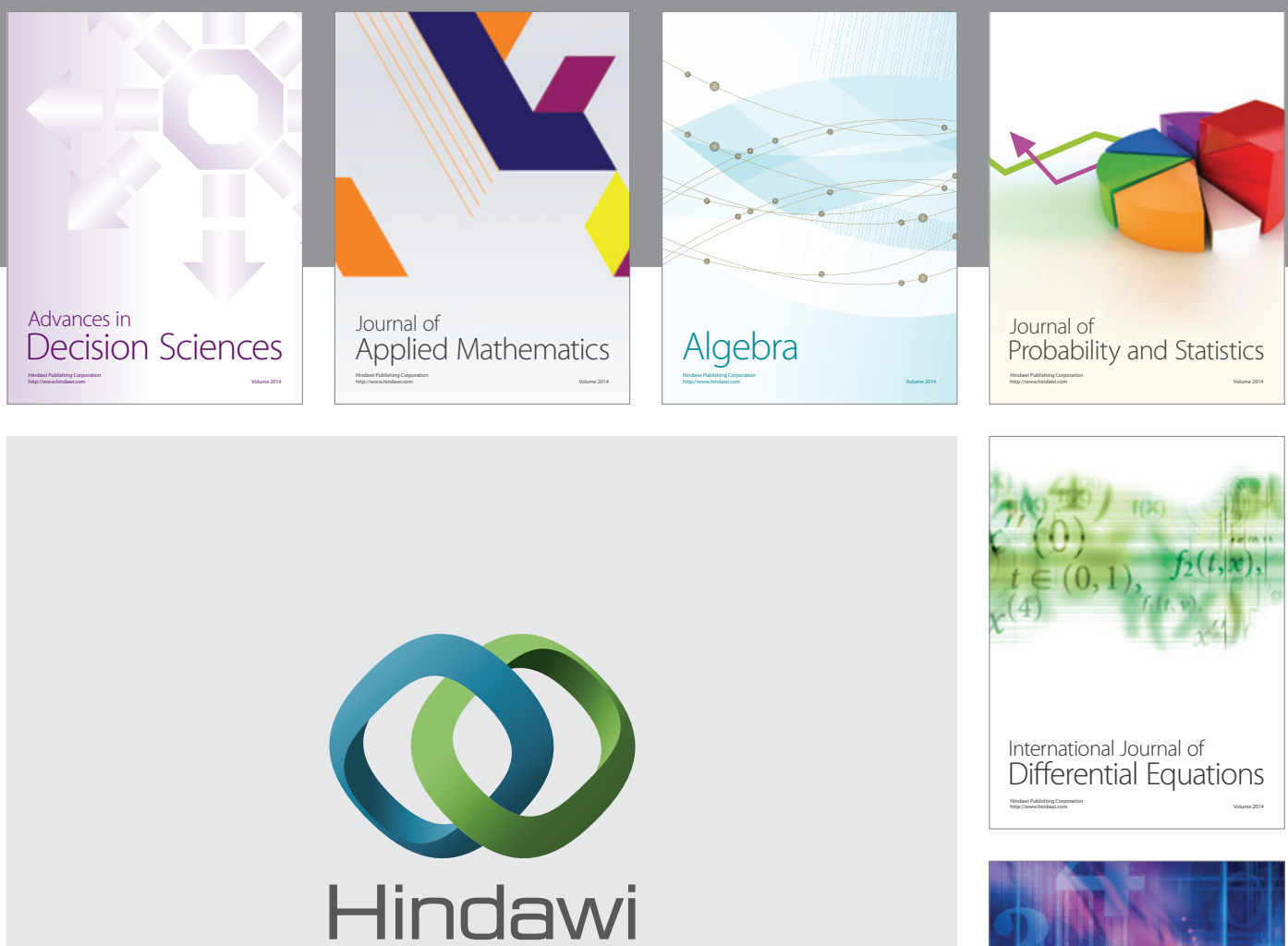

Submit your manuscripts at http://www.hindawi.com
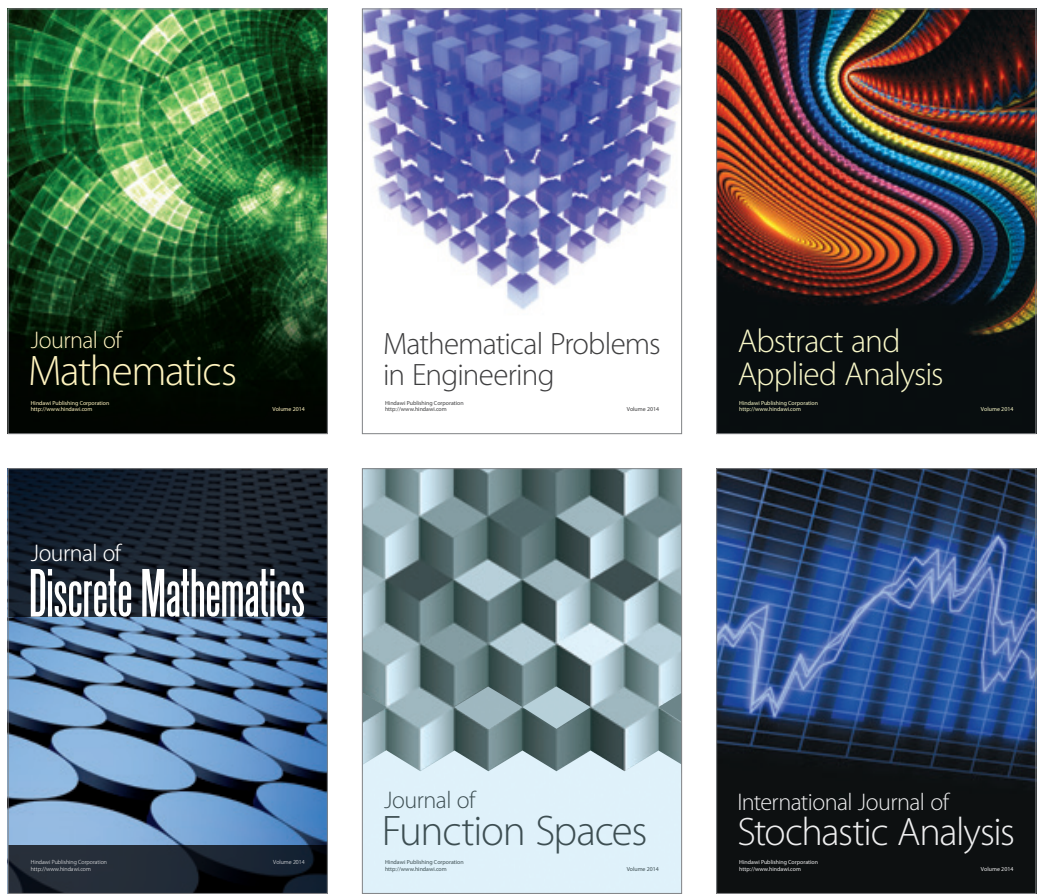

Journal of

Function Spaces

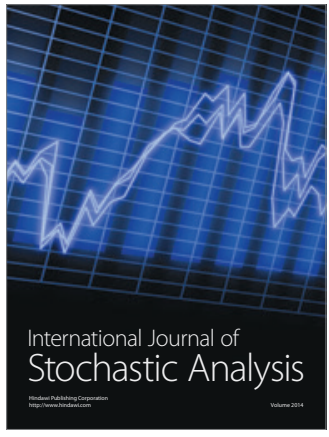

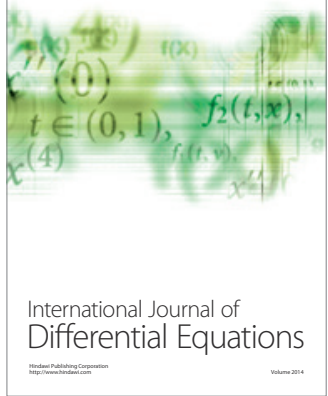
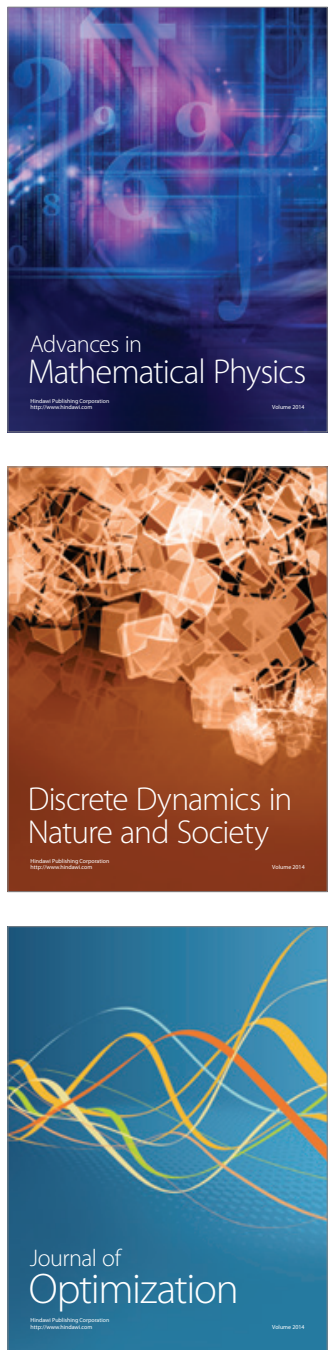